\title{
Initial transcribed region sequences influence the composition and functional properties of the bacterial elongation complex
}

\author{
Padraig Deighan, ${ }^{1}$ Chirangini Pukhrambam, ${ }^{2}$ Bryce E. Nickels, ${ }^{2}$ and Ann Hochschild ${ }^{1,3}$ \\ ${ }^{1}$ Department of Microbiology and Molecular Genetics, Harvard Medical School, Boston, Massachusetts 02115, USA; \\ ${ }^{2}$ Department of Genetics, Waksman Institute, Rutgers University, Piscataway, New Jersey 08854, USA
}

\begin{abstract}
The bacterial RNA polymerase (RNAP) holoenzyme consists of a catalytic core enzyme $\left(\alpha_{2} \beta \beta\right.$ ' $\left.\omega\right)$ in complex with a $\sigma$ factor that is essential for promoter recognition and transcription initiation. During early elongation, the stability of interactions between $\sigma$ and the remainder of the transcription complex decreases. Nevertheless, there is no mechanistic requirement for release of $\sigma$ upon the transition to elongation. Furthermore, $\sigma$ can remain associated with RNAP during transcription elongation and influence regulatory events that occur during transcription elongation. Here we demonstrate that promoter-like DNA sequence elements within the initial transcribed region that are known to induce early elongation pausing through sequence-specific interactions with $\sigma$ also function to increase the $\sigma$ content of downstream elongation complexes. Our findings establish $\sigma$-dependent pausing as a mechanism by which initial transcribed region sequences can influence the composition and functional properties of the transcription elongation complex over distances of at least 700 base pairs.
\end{abstract}

[Keywords: RNA polymerase; $\sigma$ factor; transcription initiation; promoter-proximal pausing; transcription elongation complex]

Supplemental material is available for this article.

Received September 10, 2010; revised version accepted November 5, 2010.

The $\sigma$ subunit of bacterial RNA polymerase (RNAP) is required for promoter-specific transcription initiation (Gross et al. 1998). When complexed with the RNAP core enzyme (subunit structure $\alpha_{2} \beta \beta^{\prime} \omega$ ), different $\sigma$ factors specify the recognition of different classes of promoters (Gruber and Gross 2003). The primary $\sigma$ factor in Escherichia coli, $\sigma^{70}$, typically directs transcription initiation from promoters defined by two conserved hexameric DNA sequence elements, termed the -10 and -35 elements for their relationship to the transcription start site (position +1 ). During the transition from transcription initiation to transcription elongation, the growth of the nascent RNA destabilizes the interaction between $\sigma^{70}$ and the RNAP core enzyme (Mekler et al. 2002; Murakami et al. 2002; Vassylyev et al. 2002; Nickels et al. 2005), but the complete release of $\sigma^{70}$ is not required for entry into the elongation phase of transcription (Ring et al. 1996; Bar-Nahum and Nudler 2001; Mukhopadhyay et al. 2001; Mooney et al. 2005). Thus, although historically defined as an initiation factor, $\sigma^{70}$ can also remain

${ }^{3}$ Corresponding author.

E-MAIL ahochschild@hms.harvard.edu; FAX (617) 738-7664.

Article is online at http://www.genesdev.org/cgi/doi/10.1101/gad.1991811. associated with the transcription elongation complex and influence the transcription process during elongation.

A functional role for $\sigma^{70}$ during elongation was first established in the context of bacteriophage $\lambda$ late gene transcription (for review, see Roberts et al. 1998). Specifically, the expression of the phage late genes under the control of the $\lambda \mathrm{Q}$ anti-terminator protein depends on a $\sigma^{70}$-dependent pause that occurs during early elongation, shortly after the $\sigma^{70}$-containing RNAP holoenzyme has escaped the late promoter $\mathrm{P}_{\mathrm{R}^{\prime}}$. Formation of this paused early elongation complex, which contains a stably bound nascent RNA that is 16 or 17 nucleotides (nt) in length, depends on an interaction between $\sigma^{70}$ and a DNA sequence element in the initial transcribed region positioned from +1 to +6 that resembles a promoter -10 element (Ring et al. 1996). Furthermore, the same module of $\sigma^{70}$ that contacts the promoter -10 element during transcription initiation $\left(\sigma^{70}\right.$ region 2$)$ contacts the -10 like element in the initial transcribed region during early elongation pausing (Ring et al. 1996). Similar -10-like initial transcribed region DNA sequence elements have been shown to induce $\sigma^{70}$-dependent pausing in the context of the late promoters of other lambdoid phages (Ring et al. 1996) and in the context of several E. coli 
promoters, including the lac, tnaA, and $\operatorname{csp} D$ promoters (Brodolin et al. 2004; Nickels et al. 2004; Hatoum and Roberts 2008). Furthermore, both bioinformatics analysis and a candidate-based approach suggest that $\sim 20 \%$ of all E. coli promoters may carry $\sigma^{70}$-dependent pause-inducing sequence elements in their initial transcribed regions (Nickels et al. 2004; Hatoum and Roberts 2008).

Here we establish a new function for initial transcribed region $\sigma^{70}$-dependent pause elements: to modify the functional properties of downstream elongation complexes by increasing their $\sigma^{70}$ content. In particular, we show that an elongation complex that encounters a $\sigma^{70}$-dependent pause element in the initial transcribed region is more likely to retain $\sigma^{70}$, and thus is more likely to pause at a second $\sigma^{70}$-dependent pause element positioned downstream. We further demonstrate that the effect of a promoter-proximal $\sigma^{70}$-dependent pause element on the $\sigma^{70}$ content of downstream elongation complexes can be detected over distances of at least 700 base pairs (bp). Our findings establish $\sigma^{70}$-dependent pausing as a mechanism whereby initial transcribed region sequences can influence the composition and functional properties of the elongation complex throughout a transcription unit. Unlike cis-acting sequences that function to recruit transacting factors to the elongation complex, these promoterlike initial transcribed region sequences functionally alter the transcription complex by inhibiting loss of an RNAP subunit. We propose that engagement of a promoter-proximal $\sigma^{70}$-dependent pause element increases the $\sigma^{70}$ content of downstream elongation complexes by stabilizing the association of $\sigma^{70}$ with the early elongation complex during a series of critical nucleotide addition steps when the probability of $\sigma^{70}$ release is relatively high.

\section{Results}

\section{Preliminary considerations}

Biophysical characterization of artificially stalled transcription complexes indicates that the association of $\sigma^{70}$ with the early elongation complex is stabilized by interactions between $\sigma^{70}$ and a pause-inducing sequence element in the initial transcribed region. Specifically, ensemble and single-molecule fluorescence resonance energy transfer (FRET) measurements of the $\sigma^{70}$ content of elongation complexes halted during early elongation indicate that a pause-inducing sequence element positioned from +1 to +6 (like that at $\lambda \mathrm{P}_{\mathrm{R}^{\prime}}$ ) functions to stabilize the association of $\sigma^{70}$ with stalled elongation complexes containing nascent RNAs 11-15 nt in length (Nickels et al. 2004; Kapanidis et al. 2005). However, conflicting results have been obtained regarding whether the presence of a $\sigma^{70}$ dependent pause-inducing sequence element in the initial transcribed region increases the proportion of elongation complexes that contain $\sigma^{70}$ at positions downstream from the pause site. Ensemble FRET results suggest that a $\sigma^{70}$-dependent pause-inducing sequence element in the initial transcribed region increases the $\sigma^{70}$ content of elongation complexes halted at position +50 (Mukhopadhyay et al. 2001; Nickels et al. 2004), but single-molecule FRET results do not (Kapanidis et al. 2005). Given the conflicting evidence obtained from these prior biophysical studies of halted elongation complexes, we sought to investigate whether the presence of a $\sigma^{70}$-dependent pause-inducing sequence element in the initial transcribed region influences the $\sigma^{70}$ content of downstream elongation complexes using approaches that would enable us to monitor the $\sigma^{70}$ content of actively transcribing complexes.

\section{Presence of $\sigma^{70}$-dependent pause element in the initial transcribed region increases the $\sigma^{70}$ content of downstream elongation complexes in vitro}

To determine whether the presence of a pause-inducing promoter -10-like element in the initial transcribed region increases the proportion of elongation complexes that contain $\sigma^{70}$ at downstream positions, we took advantage of prior in vitro observations indicating that, in contrast with promoter-proximal -10-like elements (i.e., those that extend no further than approximately +15 relative to the transcription start site), promoter-distal -10-like elements can induce efficient pausing only when high concentrations $(1 \mu \mathrm{M})$ of free $\sigma^{70}$ are present (Mooney and Landick 2003; Mooney et al. 2005; Sevostyanova et al. 2008). These findings suggest that efficient engagement of promoter-distal $\sigma^{70}$-dependent pause elements requires that free $\sigma^{70}$ rebind the elongation complex, likely because the majority of complexes lose $\sigma^{70}$ during the earliest phase of elongation (Mooney and Landick 2003). We therefore sought to determine whether the presence of a promoter -10-like element in the initial transcribed region could inhibit $\sigma^{70}$ loss during the earliest phase of elongation and enable $\sigma^{70}$-dependent pausing at a promoter-distal site in the absence of high concentrations of free $\sigma^{70}$.

We performed a series of in vitro transcription assays using three templates (Fig. 1). The first template (template \#1) carried both a promoter-proximal $\sigma^{70}$-dependent

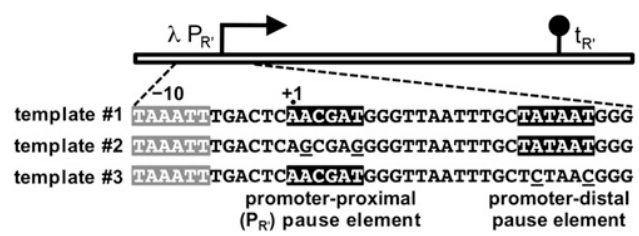

Figure 1. Schematic depiction and relevant sequence of the in vitro transcription templates. The -10 element of the $\lambda P_{R^{\prime}}$ promoter is highlighted in gray. The promoter-proximal $\lambda P_{R^{\prime}}-10-$ like pause-inducing element (positioned between +1 and +6 ) and the promoter-distal pause-inducing element (consensus -10 hexamer positioned between +19 and +24 ) are highlighted in black. The distal pause element also includes a TG dinucleotide upstream of the -10 hexamer, and thus is a consensus extended -10 element. The mutations that disrupt the promoter-proximal pause element on template \#2 and the mutations that disrupt the promoter-distal pause element on template \#3 are underlined. Terminator $t_{R^{\prime}}$ (black lollipop) is positioned to terminate transcription at $\sim 116 \mathrm{nt}$. 
pause-inducing sequence element and a promoter-distal $\sigma^{70}$-dependent pause-inducing sequence element. In particular, template \#1 carried the well-characterized pause element associated with the $\lambda$ late promoter $\left(\lambda P_{R^{\prime}}\right)$ positioned between +1 and +6 , as well as a consensus promoter -10 element positioned between +19 and +24 . The second template (template \#2) carried two base pair substitutions in the promoter-proximal pause element $(A+2 G / T+6 G)$ that destabilize interactions with $\sigma^{70}$ and eliminate $\sigma^{70}$-dependent pausing (Ring and Roberts 1994). The third template (template \#3) carried two base pair substitutions in the promoter-distal pause element $(\mathrm{A}+20 \mathrm{C} / \mathrm{T}+24 \mathrm{C})$; these substitutions were predicted to destabilize interactions with $\sigma^{70}$ and eliminate $\sigma^{70}$-dependent pausing at the promoter-distal site.

When single-round transcription time-course reactions were performed with low concentrations of $\sigma^{70}$ (30 nM, a twofold molar excess over RNAP core) using template $\# 1$, the expected 16- and 17-nt $\lambda P_{R^{\prime}}$ pause species, which appeared early in the time course and decayed over time, were observed (Fig. 2A). In addition to these 16- and 17-nt RNA transcripts, less-prominent 35- to 37-nt RNA transcripts were observed throughout the time course (Fig. 2A). Strikingly, when the transcription reactions were performed using template \#2, which carries mutations in the promoter-proximal pause element, these 35- to 37-nt RNA transcripts were not detected, consistent with the idea that the presence of a $\sigma^{70}$-dependent pause element in the initial transcribed region can facilitate $\sigma^{70}$-dependent pausing at promoter-distal sites. Several lines of evidence confirmed that the 35- to 37-nt RNA transcripts are, in fact, the result of $\sigma^{70}$-dependent pausing at the promoter-distal site. First, the promoter-distal pause element is displaced by 18 bp relative to the position of the promoter-proximal pause element (register +19 compared with register +1 ), and the 35 - to 37-nt RNA transcripts are $\sim 18 \mathrm{nt}$ longer than the paused species that result from engagement of the promoter-proximal pause element. Second, the 35- to 37-nt RNA species are absent when reactions are performed using template \#3, which carries mutations in the promoter-distal pause element (Fig. 2A). Third, production of the 35- to 37-nt RNA species (like production of the 16- and 17-nt RNA species) is reduced when reactions are done using template \#1 in the presence of RNAP holoenzyme reconstituted with a well-characterized $\sigma^{70}$ mutant $\left(\sigma^{70} \mathrm{~L} 402 \mathrm{~F}\right)$ that is deficient in $\sigma^{70}$-dependent pausing (Fig. 2B; Ko et al. 1998). Fourth, the 35- to 37-nt RNA species are sensitive to the transcript cleavage factor GreB (Fig. 2B), a hallmark of previously characterized $\sigma^{70}$-dependent pause species (Marr and Roberts 2000). We therefore conclude that the presence of a promoter-proximal $\sigma^{70}$-dependent pause element increases the likelihood that $\sigma^{70}$-dependent pausing will occur at a promoter-distal site in vitro.

To test the hypothesis that the presence of a promoterproximal pause element enables promoter-distal pausing by inhibiting the loss of $\sigma^{70}$ from the early elongation complex, we asked whether the addition of excess $\sigma^{70}$ could restore promoter-distal $\sigma^{70}$-dependent pausing on template \#2. Specifically, we performed the transcription reactions in the presence of $1 \mu M \sigma^{70}$ (a 67-fold molar excess over RNAP core) and found that the abundance of the 35- to 37-nt RNA species generated using template \#2 increased over their abundance when the reactions were performed with low concentrations of $\sigma^{70}$ (Fig. 2C,D); thus, the abundance of the 35- to 37-nt RNAs produced from template \#2 at high concentrations of $\sigma^{70}$ and from template \#1 at low concentrations of $\sigma^{70}$ were approximately the same (Fig. 2D, right). Furthermore, the addition of high concentrations of $\sigma^{70}$ also increased the abundance of the 35- to 37-nt RNA species generated in reactions performed with template \#1, but did not result in the appearance of these species with template \#3 (Fig. $2 \mathrm{C}, \mathrm{D})$. We conclude that the presence of a $\sigma^{70}$-dependent pause element in the initial transcribed region facilitates $\sigma^{70}$-dependent pausing at the promoter-distal site in vitro by inhibiting loss of $\sigma^{70}$ during the earliest phase of elongation.

Presence of $\sigma^{70}$-dependent pause element in the initial transcribed region increases the $\sigma^{70}$ content of downstream elongation complexes in vivo

Next, we sought to determine whether the presence of a promoter -10-like element in the initial transcribed region could inhibit $\sigma^{70}$ loss during the earliest phase of elongation and enable $\sigma^{70}$-dependent pausing at a promoter-distal site in vivo. To do this, we introduced a multicopy plasmid vector carrying template \#1, \#2, or \#3 into E. coli cells. We then isolated total RNA from the cells and detected RNA transcripts generated in vivo from these templates using hybridization with radiolabeled locked nucleic acid (LNA) probes (Válóczi et al. 2004). We identified transcripts associated with $\sigma^{70}$-dependent pausing in vivo based on a comparison with transcripts generated during in vitro transcription reactions.

We first sought to identify RNA transcripts produced from templates \#1 and \#3 in vivo using an LNA probe complementary in sequence to nucleotides +1 to +15 (Fig. 3A), which enabled the detection of RNA transcripts associated with promoter-proximal $\sigma^{70}$-dependent pausing (the 16- and 17-nt species) and RNA transcripts associated with promoter-distal $\sigma^{70}$-dependent pausing (the 35- to 37-nt species) (Fig. 3B, lane 1). (Note that the +1 to +15 probe is not fully complementary in sequence to transcripts produced from template \#2, which bears mutations at positions +2 and +6 , within the promoterproximal pause element.) With the +1 to +15 probe, we detected RNA transcripts associated with promoter-proximal $\sigma^{70}$-dependent pausing in RNA samples isolated from cells carrying either template \#1 or template \#3 (Fig. 3B). In contrast, RNA transcripts associated with promoter-distal $\sigma^{70}$-dependent pausing were detected only in RNA samples isolated from cells carrying template \#1, which carries an intact promoter-distal pause element (Fig. 3B).

We next determined the effect of mutating the promoter-proximal pause element on promoter-distal $\sigma^{70}$ dependent pausing in vivo. Thus, we compared the 
Deighan et al.

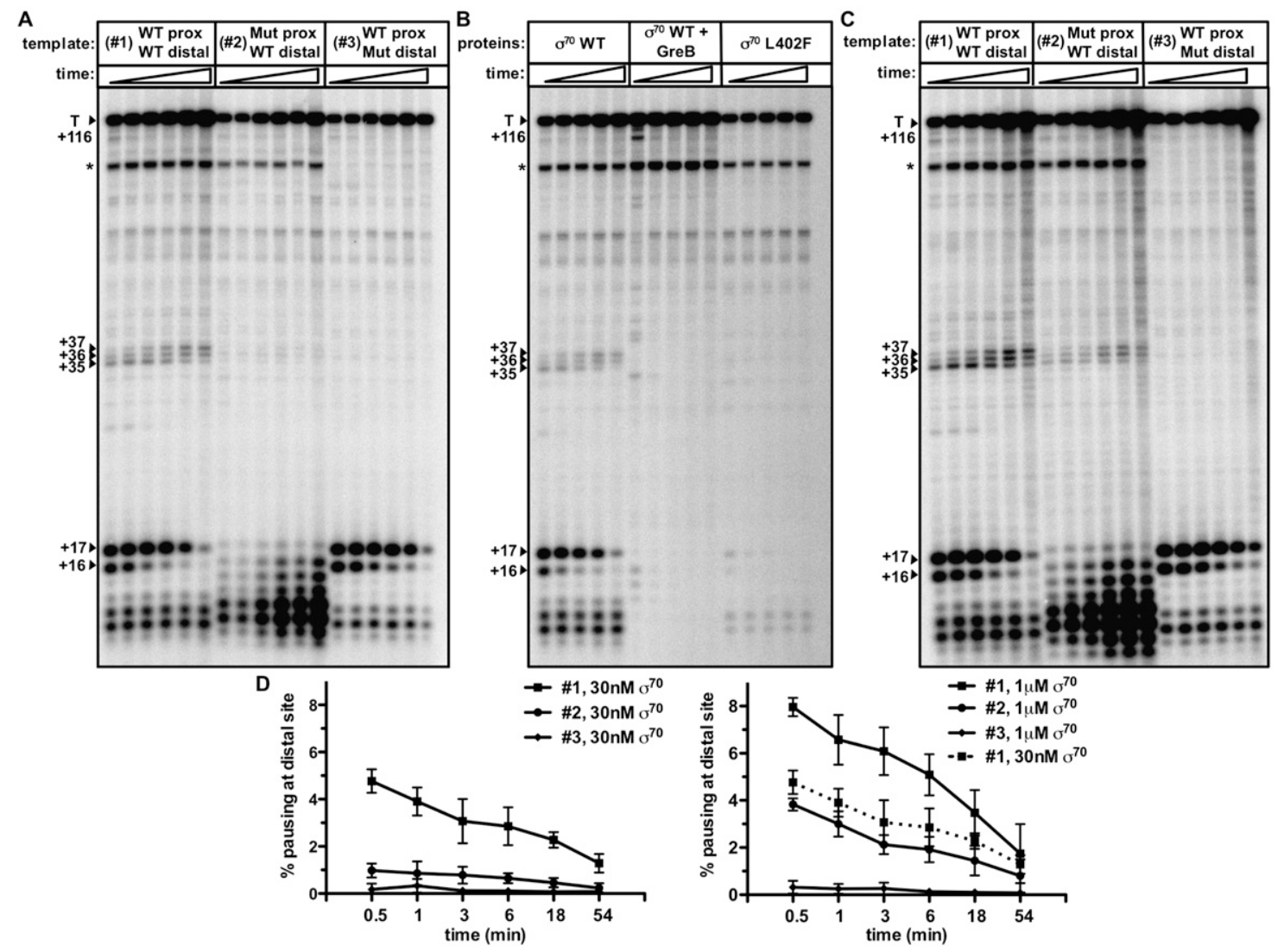

Figure 2. A promoter -10-like element in the initial transcribed region inhibits $\sigma^{70}$ loss during early elongation and enables $\sigma^{70}$ dependent pausing at a promoter-distal site in vitro. (A) Single-round in vitro transcription reactions performed with RNAP holoenzyme (15 $\mathrm{nM}$ core reconstituted with $\left.30 \mathrm{nM} \sigma^{70}\right)$ and three different templates $(\# 1$, \#2, and \#3, as depicted in Fig. 1) bearing the indicated wild-type (WT) or mutant (Mut) promoter-proximal and promoter-distal pause elements. The reactions were performed as a time course with samples taken $0.5,1,3,6,18$, and 54 min after transcription was initiated. RNA species that reflect $\sigma^{70}$-dependent pausing at the promoter-proximal element $(+16$ and +17$)$ or the promoter-distal element $(+35,+36$, and +37$)$ are indicated. The 35 - to 37 nt RNA species, which were annotated by comparison with a reaction done in the presence of a chain-terminating nucleotide, were also detected when the RNA was end-labeled with $\left[\gamma^{3}{ }^{32} \mathrm{P}\right] \mathrm{ATP}$, indicating that they are not cleavage products derived from an internal portion of the RNA (Supplemental Fig. S1). We note the accumulation of abortive RNA products in reactions performed with template \#2, which we attribute to the two base pair substitutions in the initial transcribed region; initial transcribed region alterations can have dramatic effects on abortive transcript production (Hsu et al. 2003; Chander et al. 2007). The $\sim 116$-nt terminated transcript (T) is indicated, and the asterisk $(*)$ indicates a shorter terminated transcript that is the result of transcription initiating under the control of the promoter-distal extended -10 element. $(B)$ Single-round in vitro transcription reactions performed using template \#1 (wild-type promoter-proximal and promoter-distal pause elements) with RNAP holoenzyme ( $15 \mathrm{nM}$ core reconstituted with either $30 \mathrm{nM} \sigma^{70}$ wild

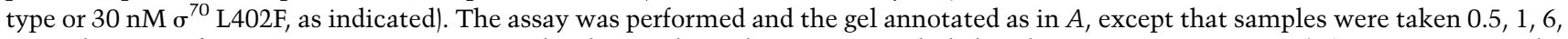
18 , and $54 \mathrm{~min}$ after transcription was initiated. Where indicated, GreB was included in the reactions at $100 \mathrm{nM}$. $(C)$ As in $A$, except the assay was performed with RNAP holoenzyme that was reconstituted with $1 \mu \mathrm{M} \sigma^{70}$. (D) Graph showing the percentage of $\sigma^{70}$ dependent pausing detected at the promoter-distal site [distal pause signal/(distal pause signal + terminated signal)] for reactions performed in the presence of $30 \mathrm{nM} \sigma^{70}$ (left graph) as in $A$, or $1 \mu \mathrm{M} \sigma^{70}$ (right graph) as in C. Plotted on the graphs are the mean and SD of three independent experiments. In the right graph, we show that the percent of pausing detected at the distal site on template \#2 in the presence of $1 \mu \mathrm{M} \sigma^{70}$ was approximately the same as that detected at the distal site on template \#1 in the presence of low concentrations (30 nM) of $\sigma^{70}$ (dotted line).

abundance of transcripts associated with promoter-distal pausing produced from template \#1 (which carries an intact promoter-proximal pause element) with the abundance of transcripts associated with promoter-distal pausing produced from template \#2 (which carries an inactivated promoter-proximal pause element). Because of the mutations present on template \#2 (at positions +2 and +6 ), we used an LNA probe complementary in sequence to nucleotides +7 to +19 for this analysis (Fig. 3A). In contrast to the +1 to +15 probe, which enabled detection of transcripts associated with promoter-proximal and promoter-distal pausing, the +7 to +19 probe enabled detection only of transcripts associated with promoterdistal $\sigma^{70}$-dependent pausing (the 35 - to 37 -nt species) (Fig. 3C). With the +7 to +19 probe, we again detected the promoter-distal 35- to 37-nt pause species in RNA samples isolated from cells carrying template \#1 and did not detect these 35- to 37-nt species when the promoter-distal 
A

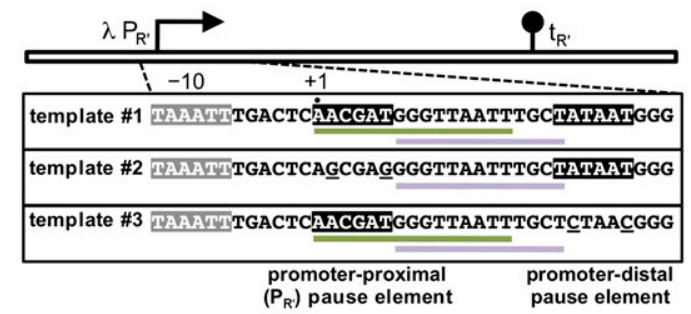

B

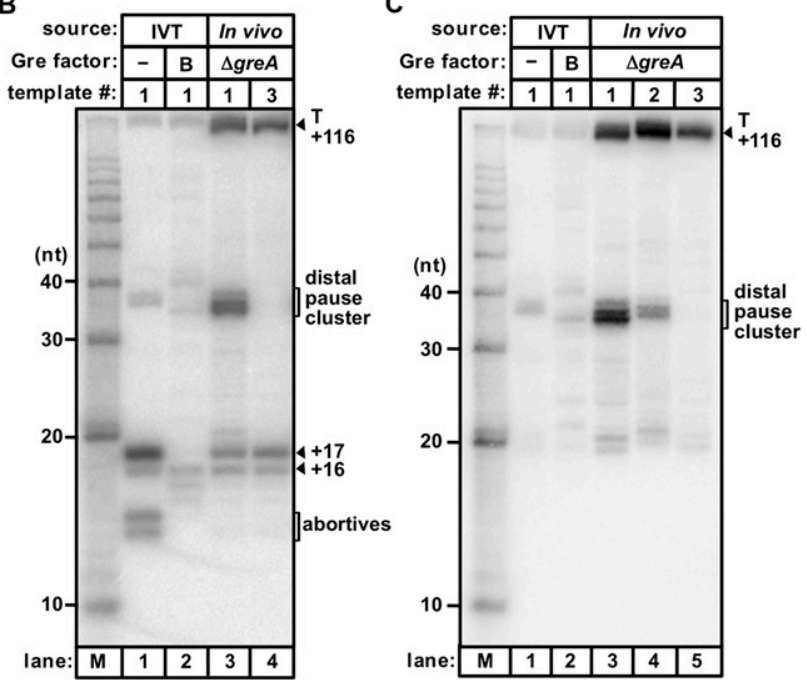

Figure 3. A promoter -10 -like element in the initial transcribed region facilitates $\sigma^{70}$-dependent pausing at a promoterdistal site in vivo. (A) Schematic of templates and LNA probes. Templates \#1, \#2, and \#3 are depicted as in Figure 1. Sequences complementary to the +1 to +15 probe (green line) or the +7 to +19 probe (lavender line) are indicated. $(B, C)$ Detection of RNA transcripts with the +1 to +15 probe $(B)$ or the +7 to +19 probe (C). The in vivo generated RNA samples isolated from a $\Delta$ gre $A$ strain to facilitate the detection of pause species (Marr and Roberts 2000) were loaded alongside transcripts generated in nonradioactive reactions performed in vitro (IVT) using template \#1 in the absence (labeled -) or presence (labeled B) of GreB; a 10-nt RNA ladder is included (lane $M$ ). We note that in vivo generated promoter-distal pause products (distal pause cluster) include a faster-migrating species that is likely due to the action of $\mathrm{GreB}$, as this species is also present when GreB is included in the in vitro transcription reaction (see also Fig. 2B). A control experiment excluded the possibility that the apparent effect of the promoter-proximal pause element on the abundance of the promoter-distal pause products is due to an effect of the $P_{R^{\prime}}$ pause mutations on the efficiency of probe hybridization (Supplemental Fig. S2).

pause element was mutationally inactivated (template \#3) (Fig. 3C). Furthermore, the abundance of the 35- to 37-nt transcripts associated with promoter-distal pausing was reduced significantly (approximately fivefold) when the $P_{R^{\prime}}$ pause element was mutationally inactivated (template \#2) (Fig. 3C). We conclude that the presence of a $\sigma^{70}$-dependent pause element in the initial transcribed region increases the likelihood that $\sigma^{70}$-dependent pausing will occur at a promoter-distal site in vivo, presumably by inhibiting loss of $\sigma^{70}$ during the earliest phase of elongation, as was revealed by our in vitro analysis (see Fig. 2).

Presence of $\sigma^{70}$-dependent pause element in the initial transcribed region increases the $\sigma^{70}$ content

of downstream elongation complexes over distances of at least $700 \mathrm{bp}$

Our results obtained in vitro and in vivo suggest that the presence of a $\sigma^{70}$-dependent promoter-proximal pause element facilitates recognition of a more downstream pause element because it enhances the $\sigma^{70}$ content of the early elongation complex. That is, transcription elongation complexes that arrive at the promoter-distal pause element are more likely to contain $\sigma^{70}$ if they have already engaged a promoter-proximal pause element. We next used chromatin immunoprecipitation (ChIP) to determine whether the effect of a promoter-proximal $\sigma^{70}$-dependent pause element on the $\sigma^{70}$ content of downstream elongation complexes could be detected throughout a transcription unit. Using cells containing a single-copy $\mathrm{P}_{\mathrm{R}^{\prime}}-1 a c Z$ fusion with either the wild-type pause element or a mutationally inactivated pause element $(A+2 G / T+6 G)$, we assessed the occupancy of both the $\beta$ subunit and the $\sigma^{70}$ subunit of RNAP across each transcription unit. To do this, we determined the fold enrichment of the promoter/pause region $(-92$ to +45$)$ and two downstream regions (centered at positions +339 and +694 ) in both the $\beta$ - and $\sigma^{70}$-immunoprecipitated material relative to a nontranscribed region of the DNA /within the $b g l$ operon). To ensure that promoter-bound transcription complexes did not contribute to the immunoprecipitation of downstream DNA (due to the difficulty of shearing the DNA to a sufficiently small average fragment length), we separated promoter DNA from downstream DNA using restriction enzymes (Fig. 4A; see also the Materials and Methods).

To compare the $\sigma^{70}$ content of elongation complexes on the two fusion constructs $\left(\mathrm{P}_{\mathrm{R}^{\prime}}-1 a c Z\right.$ containing the wildtype or mutated pause element), we calculated fold enrichment ratios $\left(\sigma^{70} / \beta\right)$ by dividing the fold enrichment of a given DNA region in the $\sigma^{70}$-immunoprecipitated material by the fold enrichment of the same region in the $\beta$-immunoprecipitated material. Although we observed a general decrease in the $\sigma^{70}$ content of elongation complexes with increasing distance from the promoter on both templates (Fig. 4B), we found that the $\sigma^{70}$ content of elongation complexes in both the +339 and +694 regions was approximately twofold higher when the $\mathrm{P}_{\mathrm{R}^{\prime}}$ pause element was intact (see Fig. 4B,D). In contrast, when the ChIP assays were done in strains containing chromosomally encoded $\sigma^{70} \mathrm{~L} 402 \mathrm{~F}$, which is deficient in $\sigma^{70}$-dependent pausing (see Fig. $2 B$ ), the fold enrichment $\left(\sigma^{70} / \beta\right)$ ratios were the same within error for each downstream region of the DNA regardless of whether the template carried a wild-type or mutated pause element (Fig. 4C,D). We conclude, therefore, that the presence of a functional $\sigma^{70}$-dependent pause element in the initial transcribed region enhances the wild-type $\sigma^{70}$ content of downstream transcription elongation complexes, and that this effect can be detected over at least $700 \mathrm{bp}$. 
Two control experiments confirmed that the association of $\sigma^{70}$ (and $\beta$ ) with downstream DNA was dependent on transcription initiating from promoter $\mathrm{P}_{\mathrm{R}^{\prime}}$. First, neither downstream DNA region was enriched above background in either the $\beta$ - or the $\sigma^{70}$-immunoprecipitated material when $\mathrm{P}_{\mathrm{R}^{\prime}}$ was inactivated (as confirmed by the results of parallel $\beta$-galactosidase assays) on the template with the intact pause element (Fig. 4D). Second, the fold enrichment values for the promoter region were high, whereas the fold enrichment values for the downstream DNA regions dropped to background or near background in both the $\beta$ - and the $\sigma^{70}$-immunoprecipitated samples when the cells were pretreated with rifampicin, which traps RNAP at the promoter (Fig. 4D; Herring et al. 2005).

To explore the generality of these observations, we tested the effect of another promoter-proximal $\sigma^{70}$-dependent pause element, which associated with the late promoter of phage 82 . Displaced by $8 \mathrm{bp}$ relative to the position of the $\lambda \mathrm{P}_{\mathrm{R}^{\prime}}$ pause element, the 82 pause element induces pausing at a nascent RNA length of $\sim 25 \mathrm{nt}$ (Goliger and Roberts 1989), as compared with 16-17 nt in the case of the $\lambda$ pause element. Using a pair of $82 \mathrm{P}_{\mathrm{R}^{\prime-}}$ lac $Z$ fusion constructs (bearing the wild-type or a mutated +25 pause element), we again used ChIP to examine the $\beta$ and $\sigma^{70}$ occupancies within the promoter/pause region and two downstream regions (centered at positions +356

A

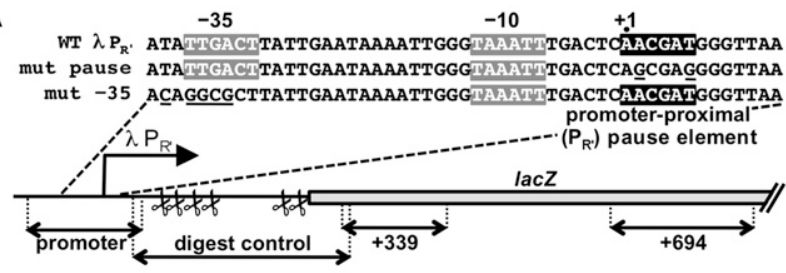

B

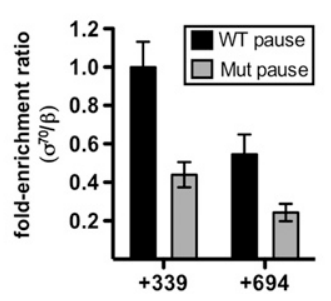

C

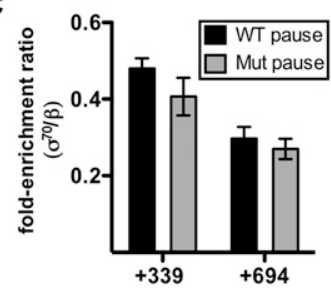

D

\begin{tabular}{|c|c|c|c|c|c|}
\hline \multirow{2}{*}{ Reporter } & \multirow{2}{*}{ MU } & \multirow{2}{*}{ ChIP } & \multicolumn{3}{|c|}{ fold enrichment over bgl } \\
\hline & & & promoter & +339 & +694 \\
\hline$\lambda \mathrm{P}_{\mathrm{R}^{-}}-\mathrm{ac} Z$ & $1404(53)$ & $\begin{array}{c}\beta \\
\sigma^{70}\end{array}$ & $\begin{array}{r}33(3.3) \\
232(8.4)\end{array}$ & $\begin{array}{l}6.4(0.6) \\
6.4(0.3)\end{array}$ & $\begin{array}{l}4.9(0.4) \\
2.6(0.3)\end{array}$ \\
\hline 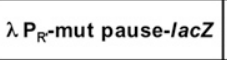 & 2483 (191) & $\underset{\sigma^{70}}{\beta}$ & $\begin{array}{l}55(5.6) \\
127(11)\end{array}$ & $\begin{array}{r}22(2.1) \\
9.7(0.8)\end{array}$ & $\begin{array}{r}15(1.2) \\
3.7(0.5)\end{array}$ \\
\hline$\lambda \mathrm{P}_{\mathrm{R}^{-}}$-mut $-35-/ a c Z$ & $30(1)$ & $\begin{array}{c}\beta \\
\sigma^{70}\end{array}$ & $\begin{array}{l}1.4(0.2) \\
2.8(0.6)\end{array}$ & $\begin{array}{l}0.7(0.1) \\
0.9(0.1)\end{array}$ & $\begin{array}{l}0.8(0.1) \\
1.1(0.1)\end{array}$ \\
\hline $\begin{aligned} & \lambda \mathrm{P}_{\mathrm{R}^{-}-\mathrm{acZ} Z} \\
&+ \text { rifampicin }\end{aligned}$ & $1348(65)$ & $\begin{array}{c}\beta \\
\sigma^{70}\end{array}$ & $\begin{array}{l}70(3.2) \\
88(3.1)\end{array}$ & $\begin{array}{l}1.5(0.1) \\
1.1(0.1)\end{array}$ & $\begin{array}{l}1.1(0.1) \\
0.9(0.1)\end{array}$ \\
\hline $\begin{array}{r}\lambda \mathrm{P}_{\mathrm{R}} \text {-mut pause-lacz } \\
+ \text { rifampicin }\end{array}$ & $2276(145)$ & $\begin{array}{c}\beta \\
\sigma^{70}\end{array}$ & $\begin{array}{l}72(6.0) \\
81(4.1)\end{array}$ & $\begin{array}{l}2.9(0.2) \\
1.7(0.1)\end{array}$ & $\begin{array}{l}1.3(0.1) \\
1.0(0.1)\end{array}$ \\
\hline $\begin{array}{r}\lambda \mathrm{P}_{\mathrm{R}^{-}}-/ a c Z \\
r p o D-L 402 F\end{array}$ & $2294(31)$ & $\begin{array}{c}\beta \\
\sigma^{70}\end{array}$ & $\begin{array}{l}14(0.2) \\
49(3.0)\end{array}$ & $\begin{array}{l}13(1.2) \\
6.2(0.2)\end{array}$ & $\begin{array}{r}20(1.3) \\
5.9(0.6)\end{array}$ \\
\hline $\begin{array}{r}\lambda \mathrm{P}_{\mathrm{R}}-\text { mut pause-lacZ } \\
r p o D-L 402 \mathrm{~F}\end{array}$ & $2691(96)$ & $\begin{array}{c}\beta \\
\sigma^{70}\end{array}$ & $\begin{array}{l}12(0.9) \\
50(4.8)\end{array}$ & $\begin{array}{l}15(1.0) \\
6.1(0.3)\end{array}$ & $\begin{array}{l}23(1.2) \\
6.1(0.3)\end{array}$ \\
\hline
\end{tabular}

and +511 ) (Fig. 5A). We found that the $\sigma^{70}$ content of elongation complexes was between 2.5 -fold and threefold higher in both the +356 and +511 regions when the $82 \mathrm{P}_{\mathrm{R}^{\prime}}$ pause element was intact (Fig. 5B,C). As we did in the case of the $\lambda \mathrm{P}_{\mathrm{R}^{\prime}}$ construct, we inactivated the $82 \mathrm{P}_{\mathrm{R}^{\prime}}$ promoter and confirmed that the association of $\sigma^{70}$ (and $\beta)$ with downstream DNA was dependent on transcription initiating from this promoter (Fig. 5C). We conclude that a promoter-proximal $\sigma^{70}$-dependent pause element can enhance the $\sigma^{70}$ content of downstream transcription elongation complexes when located in at least two distinct positions within the initial transcribed region.

\section{Discussion}

Here we identify $\sigma^{70}$-dependent pausing as a mechanism by which the sequence of the initial transcribed region

Figure 4. Effect of the $\lambda \mathrm{P}_{\mathrm{R}^{\prime}}$ promoter-proximal $\sigma^{70}$-dependent pause element on the $\sigma^{70}$ content of elongation complexes on a $\lambda \mathrm{P}_{\mathrm{R}^{\prime}}$-lacZ fusion. (A) Schematic depiction and relevant sequence of the single-copy $\lambda \mathrm{P}_{\mathrm{R}^{\prime}}$-lac $Z$ fusions used in the ChIP assay. The -35 and -10 elements of the $\lambda P_{R^{\prime}}$ promoter are highlighted in gray. The -10 -like pause-inducing element (positioned between +1 and +6 ) is highlighted in black. The mutations that disrupt the promoter-proximal pause element (on the template designated "mut pause") and the mutations within and upstream of the -35 element that disrupt the $\lambda \mathrm{P}_{\mathrm{R}^{\prime}}$ promoter (on the template designated "mut $-35^{\prime \prime}$ ) are underlined. The black two-headed arrows in the schematic represent the target regions (promoter, $+339,+694$, and digest control) that were amplified by real-time PCR in the ChIP assay. The scissor symbols represent the locations of the restriction enzyme recognition sites (all of which are within the digest control region and outside the other target regions) that were used to separate the $\beta$-immunoprecipitated (or $\sigma^{70}$-immunoprecipitated) promoter region from the downstream regions. The efficiency of digestion at these sites was monitored by quantitative amplification of the digest control region. $(B, C)$ Graphs showing the ChIP fold enrichment ratios $\left(\sigma^{70} / \beta\right)$ from cells containing a $\lambda P_{\mathrm{R}^{\prime}}$ lac $Z$ fusion with either the wild-type pause element (WT pause) or an inactivated pause element (Mut pause), as indicated. The $\sigma^{70} / \beta$ ratios were obtained by dividing the fold enrichment over background (bgl) of a given DNA region $(+339$ or +694$)$ in the $\sigma^{70}$-immunoprecipitated material by the fold enrichment of the same region in the $\beta$-immunoprecipitated material (see the Materials and Methods). We note that these fold enrichment ratios do not represent the absolute molar ratios of $\sigma^{70}$ and $\beta$ in the elongation complex (see the Discussion). In $B$, the cells contained wildtype $\sigma^{70}$, whereas in $C$, the cells contained chromosomally encoded $\sigma^{70}$ L402F. The means and SDs of three independent experiments are graphed. $(D)$ Fold enrichments over $b g l$ for both the $\beta$ - and $\sigma^{70}$-immunoprecipitated DNA from cells containing the templates shown in $A$ and either wild-type $\sigma^{70}$ or chromosomally encoded $\sigma^{70} \mathrm{~L} 402 \mathrm{~F}$, as indicated. The fold enrichments over bgl were determined as described in the Materials and Methods and represent the means of three independent experiments, with SDs in parentheses. These fold enrichment values were used to calculate the fold enrichment ratios plotted in $B$ and $C$. The cell cultures that were analyzed by ChIP were also assayed for $\beta$-galactosidase (before the addition of formaldehyde or rifampicin), and the results are expressed in Miller units (MU) with the SDs in parentheses. 
can influence both the composition and functional properties of downstream elongation complexes. In particular, our results demonstrate that the presence of a $\sigma^{70}$-dependent pause element in the initial transcribed region inhibits $\sigma^{70}$ loss during the earliest stage of elongation, increasing the $\sigma^{70}$ content of elongation complexes at least $700 \mathrm{bp}$ into the transcription unit. Moreover, the analysis of actively transcribing complexes in vitro and in vivo indicates that $\sigma^{70}$ is retained in a functionally competent state that permits it to engage downstream pause-inducing elements.

A model for how the presence of a promoter-proximal $\sigma^{70}$-dependent pause element influences the $\sigma^{70}$ content of downstream elongation complexes

Before providing a mechanistic explanation for the effect of a promoter-proximal $\sigma^{70}$-dependent pause element on the $\sigma^{70}$ content of downstream elongation complexes, we consider how the paused early elongation complex at $\lambda P_{R^{\prime}}$ is formed. The position of the $\lambda P_{R^{\prime}}-10$-like pause element relative to the promoter -10 element (displaced downstream by $12 \mathrm{bp}$ ) suggests that $\sigma^{70}$ might initially engage this element at a nascent RNA length of $\sim 12 \mathrm{nt}$. Thus, it was proposed that formation of the paused elongation complex at $\lambda P_{R^{\prime}}$ involves the establishment of sequence-specific interactions between $\sigma^{70}$ (region 2 in particular) and the -10-like element at a nascent RNA length of $\sim 12 \mathrm{nt}$, followed by several nucleotide addition steps during which $\sigma^{70}$ maintains contact with the pause element, until the transcript reaches a critical length (16 or $17 \mathrm{nt}$ ) at which pausing is detected (Marr and Roberts 2000). Consistent with the suggestion that $\sigma^{70}$ can maintain contact with the pause element through

A

$82 P_{\mathrm{R}^{\prime}}$ 'WT' $\frac{-35}{-10} \stackrel{+1}{+1}$ mut pause mut -10 TIGCAAACTGGATTAAAAAGCATCTATCTCATATAAATCTGATATTTYTGCCA

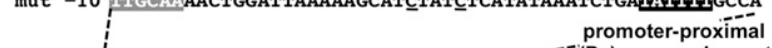

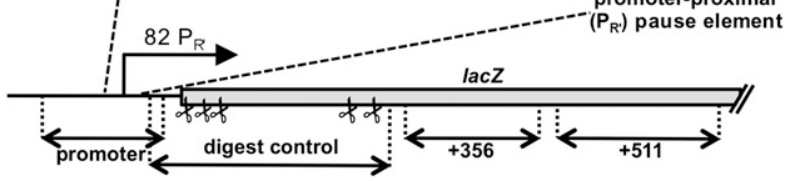

B

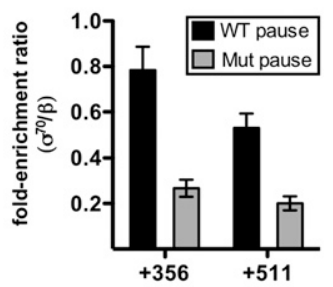

C

\begin{tabular}{|c|c|c|c|c|c|}
\hline \multirow{2}{*}{ Reporter } & \multirow{2}{*}{ MU } & \multirow{2}{*}{ ChIP } & \multicolumn{3}{|c|}{ fold enrichment over bgl } \\
& & & \multicolumn{4}{|c|}{ promoter } & +356 & +511 \\
\hline \multirow{2}{*}{$82 \mathrm{P}_{\mathrm{R}}$-lacZ } & \multirow{2}{*}{$1962(39)$} & $\beta$ & $14(0.5)$ & $3.6(0.4)$ & $3.8(0.5)$ \\
& & $\sigma^{70}$ & $92(5.9)$ & $2.8(0.1)$ & $2.0(0.1)$ \\
\hline $82 \mathrm{P}_{\mathrm{R}^{-} \text {-mut pause-lacZ }} 3032(127)$ & $\beta$ & $11(0.9)$ & $7.9(0.4)$ & $7.8(0.7)$ \\
& & $\sigma^{70}$ & $23(4.6)$ & $2.1(0.2)$ & $1.6(0.1)$ \\
\hline \multirow{2}{*}{$82 \mathrm{P}_{\mathrm{R}^{-} \text {-mut }-10-l a c Z}$} & \multirow{2}{*}{$64(2)$} & $\beta$ & $5.1(0.8)$ & $0.8(0.1)$ & $1.1(0.1)$ \\
& & $\sigma^{70}$ & $12(1.9)$ & $1.3(0.1)$ & $1.3(0.1)$ \\
\hline
\end{tabular}

several successive nucleotide addition steps, the results of ensemble FRET analysis indicate that a $\sigma^{70}$-dependent pause element positioned from +1 to +6 can stabilize the association of $\sigma^{70}$ with elongation complexes halted after the synthesis of RNA transcripts ranging in length from $11 \mathrm{nt}$ to at least $15 \mathrm{nt}$ (Nickels et al. 2004). According to the model, the nucleotide addition steps that occur while $\sigma^{70}$ maintains contact with the pause element require expansion of the transcription bubble and concomitant DNA scrunching (see Fig. 6A; Marr and Roberts 2000), as has been shown to occur in the context of promoterbound initial transcribing complexes during the process of promoter escape/abortive initiation (Kapanidis et al. 2006; Revyakin et al. 2006). In the case of initial transcription, DNA scrunching generates a "stressed intermediate" that can be relieved in two ways: Either $\sigma^{70}$ relinquishes its contacts with the promoter DNA and RNAP escapes the promoter, or the expanded bubble

Figure 5. Effect of the $82 \mathrm{P}_{\mathrm{R}^{\prime}}$ promoter-proximal $\sigma^{70}$-dependent pause element on the $\sigma^{70}$ content of elongation complexes on an $82 \mathrm{P}_{\mathrm{R}^{\prime}}$-lacZ fusion. (A) Schematic depiction and relevant sequence (see also the legend for Supplemental Fig. S3) of the single-copy $82 \mathrm{P}_{\mathrm{R}^{\prime}}$-lac $Z$ fusions used in the ChIP assay. The -35 and -10 elements of the $82 \mathrm{P}_{\mathrm{R}^{\prime}}$ promoter are highlighted in gray. The +25 pause element consists of a -10 -like hexamer (highlighted in black and positioned from +9 to +14 ) as well as an upstream TG dinucleotide, and thus is an extended -10-like element. The mutations that disrupt the +25 pause element (on

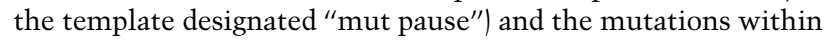
the -10 element that disrupt the $82 \mathrm{P}_{\mathrm{R}^{\prime}}$ promoter (on the template designated "mut -10 ") are underlined. An in vitro transcription assay confirmed the elimination of $\sigma^{70}$-dependent pausing on the mut pause template (Supplemental Fig. S3). The black two-headed arrows in the schematic represent the target regions (promoter, $+356,+511$, and digest control) that were amplified by real-time PCR in the ChIP assay. The scissor symbols represent the locations of the restriction enzyme recognition sites (all of which are within the digest control region and outside the other target regions) that were used to separate the $\beta$-immunoprecipitated (or $\sigma^{70}$-immunoprecipitated) promoter region from the downstream regions. The efficiency of digestion at these sites was monitored by quantitative amplification of the digest control region. (B) Graph showing the ChIP fold enrichment ratios $\left(\sigma^{70} / \beta\right)$ from cells containing a singlecopy $82 \mathrm{P}_{\mathrm{R}^{\prime}}-1$ acZ fusion with either the +25 pause element (WT pause) or a mutationally inactivated pause element (Mut pause), as indicated. The $\sigma^{70} / \beta$ ratios were obtained by dividing the fold enrichment over background (bgl) of a given DNA region $1+356$ or +511$)$ in the $\sigma^{70}$-immunoprecipitated material by the fold enrichment of the same region in the $\beta$-immunoprecipitated material (see the Materials and Methods). The means and SDs of three independent experiments are graphed. $(C)$ Fold enrichments over $b g l$ for both the $\beta$ - and $\sigma^{70}$-immunoprecipitated DNA from cells containing the templates shown in $A$. The fold enrichments over $b g l$ were determined as described in the Materials and Methods, and represent the means of three independent experiments with SDs in parentheses. These fold enrichment values were used to calculate the fold enrichment ratios plotted in $B$. The cell cultures that were analyzed by ChIP were also assayed for $\beta$-galactosidase (before the addition of formaldehyde), and the results are expressed in Miller units (MU) with the SDs in parentheses. 


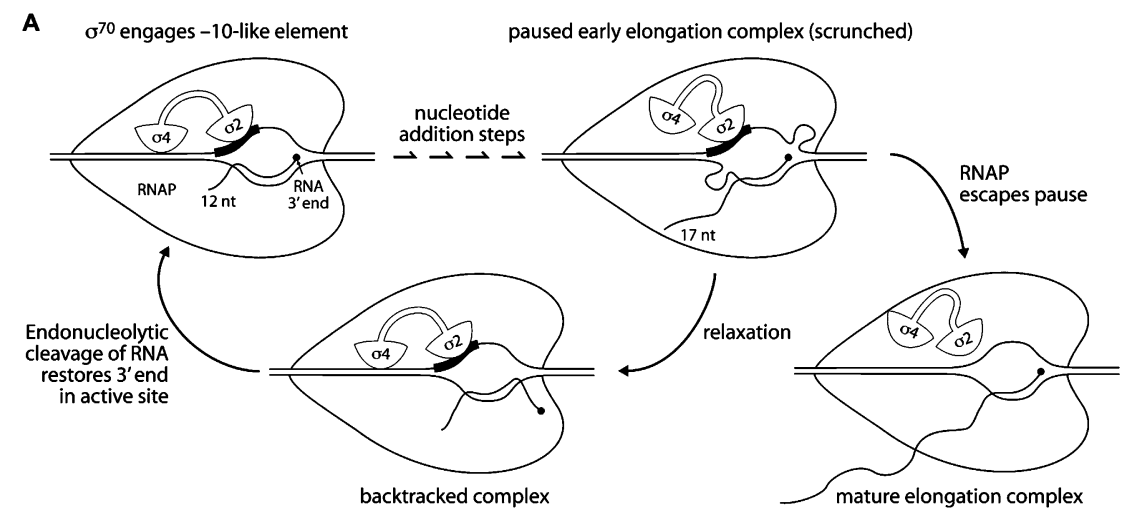

B

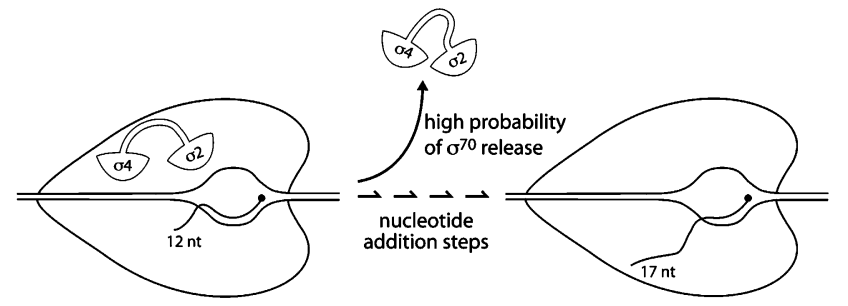

Figure 6. Model to explain how a promoterproximal $\sigma^{70}$-dependent pause element increases the $\sigma^{70}$ content of downstream elongation complexes. $(A)$ Formation of paused early elongation complex at $\lambda \mathrm{P}_{\mathrm{R}^{\prime}}$ (see also Fig. 8 in Perdue and Roberts 2010). (Top left) Domain 2 of $\sigma^{70}(\sigma 2)$ is shown engaging the promoter-proximal -10-like pause element (black rectangle) at a nascent RNA length of 12 nt. (Top right) The nascent RNA is extended to $17 \mathrm{nt}$ via a series of nucleotide addition steps that occur while $\sigma 2$ maintains contact with the pause element, requiring DNA scrunching (depicted as ssDNA loops in the transcription bubble). The scrunched complex can undergo two alternative fates: Either RNAP escapes the pause (bottom right), requiring that $\sigma 2$ relinquish contact with the pause element, or the expanded transcription bubble collapses (bottom left), generating a backtracked complex in which the catalytic center of the enzyme is no longer in register with the $3^{\prime}$ end of the RNA transcript. Endonucleolytic cleavage of the transcript (stimulated by Gre factor) (Marr and Roberts 2000) can regenerate a catalytically proficient complex with a correctly positioned RNA $3^{\prime}$ end, allowing the cycle to repeat. $(B)$ Critical window of nucleotide addition steps during which $\sigma^{70}$ release is "fast." In the absence of a promoter-proximal pause element that allows $\sigma^{70}$ to maintain stabilizing interactions with the DNA, the transcription complex releases $\sigma^{70}$ with high probability during a critical window of early nucleotide addition steps (here when the nascent RNA is extended from 12 to $17 \mathrm{nt}$ ).

collapses and an abortive transcript is released (Kapanidis et al. 2006; Revyakin et al. 2006). In the case of early elongation pausing, the strain generated by DNA scrunching can similarly be resolved in two ways: Either $\sigma^{70}$ relinquishes its contacts with the pause element and RNAP escapes the pause, or the expanded bubble collapses, in this case without release of the nascent RNA, generating a backtracked complex in which the catalytic center of the enzyme is no longer in register with the 3 ' end of the nascent transcript (Fig. 6A; Marr and Roberts 2000; Perdue and Roberts 2010).

To explain how a promoter-proximal $\sigma^{70}$-dependent pause element increases the $\sigma^{70}$ content of downstream elongation complexes, we propose that the sequencespecific interactions between $\sigma^{70}$ and the pause element stabilize the association of $\sigma^{70}$ with the early elongation complex during a "critical window" of nucleotide addition steps when the probability of $\sigma^{70}$ release is relatively high. Support for the notion of such a critical window comes from the results of both ensemble and singlemolecule FRET measurements of the $\sigma^{70}$ content of halted elongation complexes (Nickels et al. 2004; Kapanidis et al. 2005), which suggest that release of $\sigma^{70}$ is biphasic, with an initial "fast" phase that occurs after synthesis of an RNA transcript $\sim 12 \mathrm{nt}$ in length and a subsequent "slow" phase. Thus, according to our model, the interaction between $\sigma^{70}$ and an early elongation pause element stabilizes the association of $\sigma^{70}$ with the RNAP core enzyme during critical nucleotide addition steps when $\sigma^{70}$ release is "fast" (due, perhaps, to clashes between the nascent RNA and specific portions of $\sigma^{70}$ ) (for review, see
Mooney et al. 2005) and a significant fraction of the transcription complexes would otherwise release $\sigma^{70}$ (Fig. $6 \mathrm{~B})$. Our finding that the effect of an early elongation pause element on the $\sigma^{70}$ content of downstream elongation complexes was manifest when the pause element was positioned from +1 to $+6\left(\lambda P_{R^{\prime}}\right)$ or from +9 to +14 $\left(82 \mathrm{P}_{\mathrm{R}^{\prime}}\right)$ suggests that the critical window of fast $\sigma^{70}$ release might occur at different template positions, depending on the sequence context. Subsequently, as the elongation complexes leave the critical region, they appear to enter a phase where release of $\sigma^{70}$ is "slow," based on the gradual decrease in the $\sigma^{70}$ content of mature elongation complexes with increasing distance from the promoter observed in both prior studies (Raffaelle et al. 2005; Mooney et al. 2009a) and the results presented here (Figs. 4, 5).

\section{Prevalence of promoter-proximal $\sigma^{70}$-dependent pause elements in vivo}

Previous bioinformatics analysis of $\sim 650$ promoters suggested that $\sigma^{70}$-dependent promoter-proximal pause elements are associated with $\sim 20 \%$ of all promoters in $E$. coli (Nickels et al. 2004). We took advantage of a more comprehensive set of 4132 transcription start sites defined from high-throughput sequencing of the $5^{\prime}$ ends of primary RNA transcripts isolated from $E$. coli (Cho et al. 2009) to re-examine this estimate (Supplemental Table S1). The $\lambda$ pause element consists of a three of six match to the -10 element consensus sequence (TATAAT) in register +1 (where register is defined by the first base of 
the -10-like hexamer), including the highly conserved A and $\mathrm{T}$ nucleotides at positions 2 and 6 of the hexamer (McClure 1985), whereas the 82 pause element consists of a four of six match to the -10 element consensus sequence in register +9 , again including the highly conserved $\mathrm{A}$ and $\mathrm{T}$ nucleotides at positions 2 and 6 of the hexamer. For our analysis, we identified transcription units containing a three of six, four of six, five of six, or six of six match to the -10 element consensus, with an $\mathrm{A}$ and a $\mathrm{T}$ at positions 2 and 6 of the hexamer, respectively, in registers +1 to $+9 ; 40 \%$ (1654) of the transcription units contained at least a three of six match, $20 \%$ (817) contained at least a four of six match, $\sim 5 \%$ (200) contained at least a five of six match, and $\sim 0.3 \%$ (12) contained a six of six match (Table 1). Although we do not know what fraction of the 4132 transcription start sites used for this analysis are associated with a $\sigma^{70}$-dependent promoter, it is likely to be the major fraction, because most of the transcripts were present in RNA samples harvested from mid-exponential-phase cells (Cho et al. 2009).

To determine whether the initial transcribed regions of transcription units (i.e., registers +1 to +9 ) are enriched for putative $\sigma^{70}$-dependent pause elements, we computed the probabilities of finding the target sequence motifs by chance within random sequences (Table 1). Based on this analysis, we would have expected $31 \%$ of the transcription units to contain at least a three of six match, $12.5 \%$ to contain at least a four of six match, $2.7 \%$ to contain at least a five of six match, and $0.2 \%$ to contain a six of six match. This analysis therefore suggests that initial transcribed regions are enriched in $\sigma^{70}$-dependent pause elements. Consistent with this inference, we found that, when we searched for the target sequence motifs at five distinct promoter-distal positions /registers +51 to $+59,+101$ to $+109,+151$ to $+159,+201$ to +209 , and +251 to +259 ), the percentages of transcription units containing matches in each of these regions approached the percentages that were calculated based on the probabilities of finding the target sequence motifs by chance (Table 1).

\section{Functional roles for $\sigma^{70}$ during transcription elongation}

The results of prior ChIP analyses reveal unexplained differences in the $\sigma^{70}$ content of transcription elongation complexes on different transcription units (Wade and Struhl 2004; Grainger et al. 2005; Reppas et al. 2006; Mooney et al. 2009a). Our finding that the presence of a promoter-proximal $\sigma^{70}$-dependent pause element influences the $\sigma^{70}$ content of transcription complexes up to $\sim 700$ bp downstream from the transcription start site suggests that the sequence of the initial transcribed region may be a key determinant of at least some of these differences. We note that our ChIP data do not permit an estimate of the actual fractional occupancy of $\sigma^{70}$ in the elongation complex at any particular position within a transcription unit because we do not know how the cross-linking efficiencies of $\sigma^{70}$ and RNAP in a promoterbound or paused early elongation complex compare with those in an actively transcribing elongation complex.

Using an artificial construct, we demonstrated that the presence of a promoter-proximal $\sigma^{70}$-dependent pause element facilitated $\sigma^{70}$-dependent pausing at a promoterdistal pause site both in vitro and in vivo. Interestingly, promoter-distal $\sigma^{70}$-dependent pausing has been proposed to occur at a cluster of sites extending as far as $\sim 100 \mathrm{bp}$ downstream from the transcription start site of the $E$. coli tnaA promoter, which also carries a $\sigma^{70}$-dependent pause element in its initial transcribed region (Hatoum and Roberts 2008). Our findings suggest that the observed promoter-distal pausing in the tna $A$ transcription unit may be influenced by the presence of the $\sigma^{70}$-dependent pause element in the initial transcribed region.

$\sigma^{70}$-dependent pausing has been shown to affect the production of full-length transcripts, presumably by modulating RNAP density within the transcription unit (see also Fig. 4D; Hatoum and Roberts 2008). Such effects on RNAP density within a transcription unit could, in principle, affect the overall elongation rate and modulate the responsiveness of RNAP to termination signals by influencing the extent of RNAP "cooperation" (Epshtein

Table 1. Enrichment of putative $\sigma^{70}$-dependent pause elements in the initial transcribed regions of E. coli transcription units

\begin{tabular}{|c|c|c|c|c|c|c|c|c|c|c|c|c|c|c|}
\hline \multirow{2}{*}{$\begin{array}{l}\text { Match to } \\
\sigma^{70} \text {-dependent } \\
\text { pause element }\end{array}$} & \multicolumn{12}{|c|}{$\begin{array}{c}\text { Number and percentage of matched } \sigma^{70} \text {-dependent pause elements in the initial } \\
\text { transcribed regions (registers }+1 \text { to }+9 \text { [ITR]) of } 4132 \text { identified transcription units } \\
\text { and in five downstream regions DR } 1-D R 5^{c}\end{array}$} & \multicolumn{2}{|c|}{$\begin{array}{l}\text { Probability of } \\
\sigma^{70} \text {-dependent pause } \\
\text { elements in randomized } \\
\text { 14-nt sequences }\end{array}$} \\
\hline & & ITR & & DR1 & & DR2 & & DR3 & & DR4 & & DR5 & $\mathrm{G} / \mathrm{C}=\mathrm{A} / \mathrm{T}$ & $\mathrm{G} / \mathrm{C}>\mathrm{A} / \mathrm{T}$ \\
\hline $\begin{array}{l}\text { At least three } \\
\text { of six }\end{array}$ & 1654 & $(40.0 \%)$ & 1546 & $(37.4 \%)$ & 1362 & $(32.9 \%)$ & 1323 & $(32.0 \%)$ & 1253 & $(30.3 \%)$ & 1248 & $(30.2 \%)$ & $32.0 \%$ & $31.0 \%$ \\
\hline $\begin{array}{l}\text { At least four } \\
\text { of six }\end{array}$ & 817 & $(19.8 \%)$ & 701 & $(16.9 \%)$ & 564 & $(13.6 \%)$ & 555 & $(13.4 \%)$ & 489 & $(11.8 \%)$ & 463 & $(11.2 \%)$ & $13.2 \%$ & $12.5 \%$ \\
\hline $\begin{array}{l}\text { At least five } \\
\text { of six }\end{array}$ & 200 & $(4.8 \%)$ & 149 & $(3.6 \%)$ & 101 & $(2.4 \%)$ & 85 & $(2.1 \%)$ & 74 & $(1.8 \%)$ & 72 & $(1.7 \%)$ & $2.9 \%$ & $2.7 \%$ \\
\hline Six of six & 12 & $(0.3 \%)$ & 7 & $(0.2 \%)$ & 4 & $(0.1 \%)$ & 5 & $(0.1 \%)$ & 4 & $(0.1 \%)$ & 3 & $(0.1 \%)$ & $0.2 \%$ & $0.2 \%$ \\
\hline
\end{tabular}

${ }^{a}$ AnnnT and at least an $X$ of six match to TATAAT.

${ }^{\mathrm{b}}$ The 4132 transcription start sites were identified by Cho et al. (2009).

${ }^{c}$ Registers +51 to +59 (DR1), +101 to +109 (DR2), +151 to +159 (DR3), +201 to +209 (DR4), and +251 to +259 (DR5).

${ }^{\mathrm{d}}$ Analysis was performed on 14-nt randomized sequences, assuming each nucleotide was used with equal efficiency (denoted G/C = $\mathrm{A} / \mathrm{T}$ ), and also using a $\mathrm{G}+\mathrm{C}$ parameter of $50.8 \%$ (denoted $\mathrm{G} / \mathrm{C}>\mathrm{A} / \mathrm{T}$ ) to reflect the $\mathrm{G}+\mathrm{C}$ content of E. coli MG1655. 
and Nudler 2003). As a component of the elongation complex, $\sigma^{70}$ could also influence transcription through mechanisms independent of its ability to induce pausing. For example, $\sigma^{70}$ could influence the accessibility of the transcription elongation complex to regulatory factors. In this regard, the $\beta^{\prime}$ coiled-coil, which binds $\sigma^{70}$ region 2 , has also been identified as a binding site for at least two elongation factors, including the highly conserved general elongation factor NusG and the NusG paralog RfaH (Belogurov et al. 2007; Mooney et al. 2009b; Nickels 2009). Essential in E. coli, NusG influences the rate of transcription elongation and functions as both a termination and an anti-termination factor (for review, see Roberts et al. 2008). As NusG is expected to compete with $\sigma^{70}$ for access to the elongation complex (Sevostyanova et al. 2008), the presence of a promoter-proximal $\sigma^{70}$ dependent pause element could potentially influence gene expression by decreasing the NusG content of downstream elongation complexes. Finally, $\sigma^{70}$ might exert a direct effect (either positive or negative) on the process of transcription termination (for example, see Arndt and Chamberlin 1988).

\section{Materials and methods}

\section{Strains and plasmids}

A list of strains and plasmids is provided in Supplemental Table S2.

\section{In vitro transcription}

His-tagged wild-type $\sigma^{70}$ and $\sigma^{70}$ L402F were purified as described (Nickels et al. 2004). E. coli RNAP core enzyme was purchased from Epicentre and GreB was a gift from J. Gelles (Brandeis University). Open complexes were formed by incubating $15 \mathrm{nM}$ RNAP (preincubated with either $30 \mathrm{nM}$ wild-type $\sigma^{70}, 30 \mathrm{nM} \mathrm{\sigma ^{70 }} \mathrm{L} 402 \mathrm{~F}$, or $1 \mu \mathrm{M}$ wild-type $\sigma^{70}$ ) with $20 \mathrm{nM}$ linear DNA template for $5 \mathrm{~min}$ at $37^{\circ} \mathrm{C}$ in transcription buffer $(20 \mathrm{mM}$ Tris- $\mathrm{HCl}$ at $\mathrm{pH}$ 8.0, $0.1 \mathrm{mM}$ EDTA, $50 \mathrm{mM} \mathrm{KCl}, 100 \mu \mathrm{g} / \mathrm{mL}$ BSA, $10 \mathrm{mM}$ DTT) plus $200 \mu \mathrm{M}$ GTP, ATP, and CTP, and $50 \mu \mathrm{M} \mathrm{UTP}$ (supplemented with $1 \mu \mathrm{Ci} / \mu \mathrm{L}\left[\alpha-{ }^{32} \mathrm{P}\right]-\mathrm{UTP}$ ). A single round of transcription was initiated by adding $4 \mathrm{mM} \mathrm{MgCl}_{2}$ and $10 \mu \mathrm{g} / \mathrm{mL}$ rifampicin. Reactions were stopped and processed at the indicated times (procedure as described in Nickels et al. 2005), and RNA transcripts were electrophoresed on $12 \%$ polyacrylamide sequencing gels. Bands were visualized by PhosphorImager, and the data were analyzed using ImageQuant software.

\section{Detection of RNA transcripts by hybridization}

ML176 cells containing plasmid pFW11tet- $\mathrm{P}_{\mathrm{R}_{-}^{\prime}+19}$, pFW11tet-

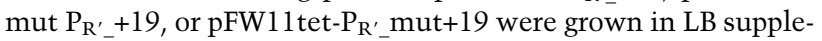
mented with tetracycline $(10 \mu \mathrm{g} / \mathrm{mL})$ to an OD600 of 0.5 . Cell suspensions ( $5 \mathrm{~mL}$ ) were mixed with RNAlater (15 mL; Ambion), collected by centrifugation (20 min at 17,000g), and resuspended in TRI Reagent ( $1 \mathrm{~mL}$; Molecular Research Center), and RNA species $<200 \mathrm{nt}$ in size were isolated using mirVana miRNA Isolation kit (Ambion). Transcripts were detected as described in Goldman et al. (2009).

\section{$\beta$-Galactosidase assays}

Reporter strain cells (in triplicate) were harvested from cultures grown at $37^{\circ} \mathrm{C}$ to mid-exponential phase $\left(\mathrm{OD}_{600}=0.5\right)$ in $\mathrm{LB}$ broth supplemented with tetracycline $(10 \mu \mathrm{g} / \mathrm{mL})$. $\beta$-Galactosidase assays were performed as described (Nickels 2009) using microtiter plates and a microtiter plate reader. Miller units were calculated as described (Nickels 2009).

\section{ChIP assay}

Cultures were grown as described for the $\beta$-galactosidase assays. Formaldehyde-induced cross-linking, cell lysis, and DNA shearing were preformed as described (Deighan and Hochschild 2007). When present, rifampicin was added to $150 \mu \mathrm{g} / \mathrm{mL}$ and cells were incubated for $20 \mathrm{~min}$ prior to addition of formaldehyde. Protein/ DNA complexes were immunoprecipitated in immunoprecipitation buffer $(50 \mathrm{mM}$ HEPES at $\mathrm{pH} 7,150 \mathrm{mM} \mathrm{NaCl}, 1 \%$ Triton $\mathrm{X}-100,0.1 \%$ sodium deoxycholate, $1 \mathrm{mM}$ PMSF) using a monoclonal antibody reactive against the $\beta$ subunit of RNAP /clone NT63, Neoclone) or a monoclonal antibody reactive against $\sigma^{70}$ (clone 2G10, Neoclone) and $20 \mu \mathrm{L}$ of protein-A/G magnetic beads (New England Biolabs). Immunoprecipitated complexes were washed once in restriction enzyme buffer 2 (New England Biolabs), and then incubated in the same buffer containing a restriction enzyme cocktail for $1 \mathrm{~h}$ at $37^{\circ} \mathrm{C}$. The restriction enzymes cleave within the "digest control region" (a region nested between the promoter region and the first downstream region) (Figs. 4A, 5A) and serve to increase the resolution of the assay by separating promoter-associated $\beta$ (or $\sigma^{70}$ )/DNA complexes from downstream regions. For ChIP experiments with the $\lambda \mathrm{P}_{\mathrm{R}^{\prime}}$ lac $Z$ fusion, the enzymes used were AciI, AhdI, HindIII, XmnI, and NdeI. For ChIP experiments with the 82

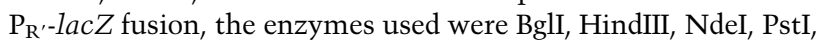
and PvuII. Complexes were then washed four times with $1 \times$ immunoprecipitation buffer and once with TE buffer $(10 \mathrm{mM}$ Tris at $\mathrm{pH} 8,1 \mathrm{mM}$ EDTA), and were resuspended in elution buffer (TE buffer containing 1\% SDS). The cross-links were reversed for $7 \mathrm{~h}$ at $65^{\circ} \mathrm{C}$, and the $\beta$ - and $\sigma^{70}$-immunoprecipitated DNAs were purified using Zymo Research spin columns.

\section{ChIP assay quantitation by real-time PCR}

For each ChIP experiment, there were five target regions of interest (Figs. 4A, 5A): the promoter region, two downstream regions within the fused lac $Z$ gene, a digest control region, and, as a background control, a region of the $b g l F$ gene within the $b g l$ operon. The chromosome-encoded $b g l$ operon is transcriptionally silent in wild-type E. coli; therefore, the analysis of $b g l$ product amplification provided an internal standard for DNA that was nonspecifically immunoprecipitated by the $\beta$ and $\sigma^{70}$ antibodies or by the protein-A/G magnetic beads. All primer pairs (purchased from IDT) (Supplemental Table S3) permitted the amplification of a single product of the predicted size with $\sim 1.93$-fold amplification per cycle using $10-\mu \mathrm{L}$ reactions containing iTaq SYBR Green Supermix with ROX (Bio-Rad) and a StepOnePlus Real-Time PCR System (Applied Biosystems). Quantitative PCRs were run in triplicate for each immunoprecipitated DNA sample, and the averaged threshold cycle $(\mathrm{Ct})$ of the amplification products was determined at a specific $\Delta \mathrm{Rn}$ [/fluorescence signal of reporter dye/fluorescence signal of passive ROX dye) - baseline]. For each $\beta$ - and $\sigma^{70}$-immunoprecipitated sample, the fold enrichment of target DNA regions over background (bgl) was calculated using the formula $1.93^{\mathrm{Ct}(\mathrm{bgl})}-\mathrm{Ct}($ target region) . All fold enrichment values represent the average of three biological replicates, and standard deviations are presented in parentheses (Figs. 4D, 5C). For the digest control regions, the fold enrichments over $b g l$ were in the range of $0.03-0.38$, indicating that promoter-bound transcription complexes did not contribute to the immunoprecipitation of downstream DNA. 


\section{Acknowledgments}

We thank J. Gelles and J.W. Roberts for helpful discussion, J. Gelles for purified GreB, and S. Dove for comments on the manuscript. We thank Pinal Kanabar (Waksman Genomics Core Facility) for analysis of initial transcribed region sequences, Peter Hochschild for computing the probabilities that random sequences contain the target sequence motifs, and Jonathan Livny for assistance with generating Supplemental Table S1. Work was supported by NIH GM44025 to A.H., and a Pew Scholars Award to B.E.N.

\section{References}

Arndt KM, Chamberlin MJ. 1988. Transcription termination in Escherichia coli. Measurement of the rate of enzyme release from Rho-independent terminators. J Mol Biol 202: 271-285.

Bar-Nahum G, Nudler E. 2001. Isolation and characterization of $\sigma^{70}$-retaining transcription elongation complexes from Escherichia coli. Cell 106: 443-451.

Belogurov GA, Vassylyeva MN, Svetlov V, Klyuyev S, Grishin NV, Vassylyev DG, Artsimovitch I. 2007. Structural basis for converting a general transcription factor into an operonspecific virulence regulator. Mol Cell 26: 117-129.

Brodolin K, Zenkin N, Mustaev A, Mamaeva D, Heumann H. 2004. The $\sigma^{70}$ subunit of RNA polymerase induces lacUV5 promoter-proximal pausing of transcription. Nat Struct Mol Biol 11: 551-557.

Chander M, Austin KM, Aye-Han N-N, Sircar P, Hsu LM. 2007. An alternate mechanism of abortive release marked by the formation of very long abortive transcripts. Biochemistry 46: 12687-12699.

Cho B-K, Zengler K, Qiu Y, Park YS, Knight EM, Barrett CL, Gao $\mathrm{Y}$, Palsson BO. 2009. The transcription unit architecture of the Escherichia coli genome. Nat Biotechnol 11: 1043-1049.

Deighan P, Hochschild A. 2007. The bacteriophage $\lambda Q$ antiterminator protein regulates late gene expression as a stable component of the transcription elongation complex. Mol Microbiol 63: 911-920.

Epshtein V, Nudler E. 2003. Cooperation between RNA polymerase molecules in transcription elongation. Science 300: 801-805.

Goldman SR, Ebright RH, Nickels BE. 2009. Direct detection of abortive RNA transcripts in vivo. Science 324: 927-928.

Goliger JA, Roberts JW. 1989. Sequences required for antitermination by phage $82 \mathrm{Q}$ protein. J Mol Biol 210: 461-471.

Grainger DC, Hurd D, Harrson M, Holdstock J, Busby SJ. 2005. Studies of the distribution of Escherichia coli cAMP-receptor protein and RNA polymerase along the E. coli chromosome. Proc Natl Acad Sci 102: 17693-17698.

Gross CA, Chan C, Dombroski A, Gruber T, Sharp M, Tupy J, Young B. 1998. The functional and regulatory roles of $\sigma$ factors in transcription. Cold Spring Harb Symp Quant Biol 63: $141-155$.

Gruber TM, Gross CA. 2003. Multiple $\sigma$ subunits and the partitioning of bacterial transcription space. Annu Rev Microbiol 57: 441-466.

Hatoum A, Roberts JW. 2008. Prevalence of RNA polymerase stalling at Escherichia coli promoters after open complex formation. Mol Microbiol 68: 17-28.

Herring CD, Raffaelle M, Allen TE, Kanin EI, Landick R, Ansari AZ, Palsson BO. 2005. Immobilization of Escherichia coli RNA polymerase and location of binding sites by use of chromatin immunoprecipitation and microarrays. I Bacteriol 187: 6166-6174.
Hsu LM, Vo NV, Kane CM, Chamberlin MJ. 2003. In vitro studies of transcript initiation by Escherichia coli RNA Polymerase. 1. RNA chain initiation, abortive initiation, and promoter escape at three bacteriophage promoters. Biochemistry 42: 3777-3786.

Kapanidis A, Margeat E, Laurence TA, Doose S, Ho SO, Mukhopadhyay J, Kortkhonjia E, Mekler V, Ebright RH, Weiss S. 2005. Retention of transcription initiation factor $\sigma^{70}$ in transcription elongation: Single-molecule analysis. $\mathrm{Mol}$ Cell 20: 347-356.

Kapanidis A, Margeat E, Ho SO, Kortkhonjia E, Weiss S, Ebright RH. 2006. Initial transcription by RNA polymerase proceeds through a DNA-scrunching mechanism. Science 314: 11441147.

Ko DC, Marr MT, Guo TS, Roberts JW. 1998. A surface of Escherichia coli $\sigma^{70}$ required for promoter function and antitermination by phage $\lambda$ Q protein. Genes Dev 12: 3276-3285.

Marr MT, Roberts JW. 2000. Function of transcription cleavage factors GreA and GreB at a regulatory pause site. Mol Cell 6: 1275-1285.

McClure WR. 1985. Mechanism and control of transcription initiation in prokaryotes. Annu Rev Biochem 54: 171-204.

Mekler V, Kortkhonjia E, Mukhopadhyay J, Knight J, Revyakin A, Kapanidis AN, Niu W, Ebright YW, Levy R, Ebright RH. 2002. Structural organization of bacterial RNA polymerase holoenzyme and the RNA polymerase-promoter open complex. Cell 108: 599-614.

Mooney RA, Landick R. 2003. Tethering a $\sigma^{70}$ to RNA polymerase reveals high in vivo activity of $\sigma$ factors and $\sigma^{70}$. dependent pausing at promoter-distal locations. Genes Dev 17: 2839-2851.

Mooney RA, Darst SA, Landick R. 2005. $\sigma$ and RNA polymerase: An on-again, off-again relationship? Mol Cell 20: 335-345.

Mooney RA, Davis SE, Peters JM, Rowland JL, Ansari AZ, Landick R. 2009a. Regulator trafficking on bacterial transcription units in vivo. Mol Cell 33: 97-108.

Mooney RA, Schweimer K, Rosch P, Gottesman M, Landick R. 2009 b. Two structurally independent domains of $E$. coli NusG create a regulatory plasticity via distinct interactions with RNA polymerase and regulators. J Mol Biol 391: 341-358.

Mukhopadhyay J, Kapanidis AN, Mekler V, Kortkhonjia E, Ebright YW, Ebright RH. 2001. Translocation of $\sigma^{70}$ with RNA polymerase during transcription: Fluorescence resonance energy transfer assay for movement relative to DNA. Cell 106: 453-463.

Murakami KS, Masuda S, Darst SA. 2002. Structural basis of transcription initiation: T. aquaticus RNA polymerase holoenzyme at $4 \AA$ resolution. Science 296: 1280-1284.

Nickels BE. 2009. Genetic assays to define and characterize protein-protein interactions involved in gene regulation. Methods 47: 53-62.

Nickels BE, Mukhopadhyay J, Garrity SJ, Ebright RH, Hochschild A. 2004. The $\sigma^{70}$ subunit of RNA polymerase mediates a promoter-proximal pause at the lac promoter. Nat Struct Mol Biol 11: 544-550.

Nickels BE, Garrity SJ, Mekler V, Minakhin L, Severinov K, Ebright RH, Hochschild A. 2005. The interaction between $\sigma^{70}$ and the $\beta$-flap of Escherichia coli RNA polymerase inhibits extension of nascent RNA during early elongation. Proc Natl Acad Sci 102: 4488-4493.

Perdue SA, Roberts JW. 2010. A backtrack-inducing sequence is an essential component of E. coli $\sigma^{70}$-dependent promoterproximal pausing. Mol Microbiol 78: 636-650.

Raffaelle M, Kanin EI, Vogt J, Burgess RR, Ansari AZ. 2005. Holoenzyme switching and stochastic release of $\sigma$ factors from RNA polymerase in vivo. Mol Cell 20: 357-366. 


\section{Deighan et al.}

Reppas NB, Wade JT, Church GM, Struhl K. 2006. The transition between transcriptional initiation and elongation in E. coli is highly variable and often rate limiting. Mol Cell 24: 747-757.

Revyakin A, Liu C, Ebright RH, Strick TR. 2006. Abortive initiation and productive initiation by RNA polymerase involve DNA scrunching. Science 314: 1139-1143.

Ring BZ, Roberts JW. 1994. Function of a nontranscribed DNA strand site in transcription elongation. Cell 78: 317-324.

Ring BZ, Yarnell WS, Roberts JW. 1996. Function of E. coli RNA polymerase $\sigma$ factor $\sigma^{70}$ in promoter-proximal pausing. Cell 86: 485-493.

Roberts JW, Yarnell W, Bartlett E, Guo J, Marr M, Ko DC, Sun H, Roberts CW. 1998. Antitermination by bacteriophage $\lambda \mathrm{Q}$ protein. Cold Spring Harb Symp Quant Biol 63: 319-325.

Roberts JW, Shankar S, Filter JJ. 2008. RNA polymerase elongation factors. Annu Rev Microbiol 62: 211-233.

Sevostyanova A, Svetlov V, Vassylyev DG, Artsimovitch I. 2008. The elongation factor $\mathrm{RfaH}$ and the initiation factor $\sigma$ bind to the same site on the transcription elongation complex. Proc Natl Acad Sci 105: 865-870.

Válóczi A, Hornyik C, Varga N, Burgyán J, Kauppinen S, Havelda Z. 2004. Sensitive and specific detection of microRNAs by northern blot analysis using LNA-modified oligonucleotide probes. Nucleic Acids Res 32: e175. doi: 10.1093/nar/gnh171.

Vassylyev DG, Sekine S, Laptenko O, Lee J, Vassylyeva MN, Borukhov S, Yokoyama S. 2002. Crystal structure of a bacterial RNA polymerase holoenzyme at $2.6 \AA$ resolution. Nature 417: 712-719.

Wade JT, Struhl K. 2004. Association of RNA polymerase with transcribed regions in Escherichia coli. Proc Natl Acad Sci 101: $17777-17782$. 


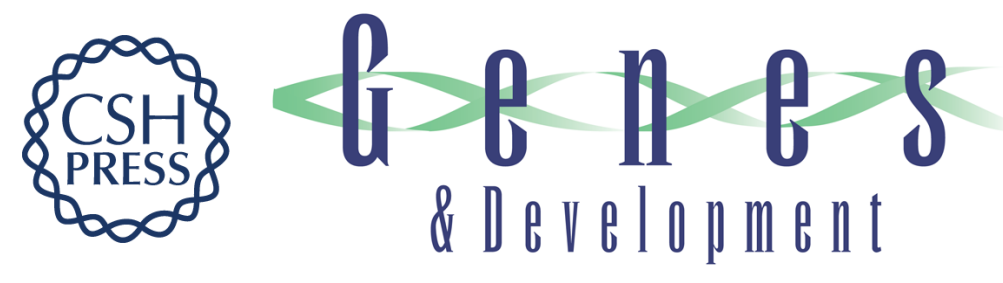

\section{Initial transcribed region sequences influence the composition and functional properties of the bacterial elongation complex}

Padraig Deighan, Chirangini Pukhrambam, Bryce E. Nickels, et al.

Genes Dev. 2011, 25:

Access the most recent version at doi:10.1101/gad.1991811

Supplemental http://genesdev.cshlp.org/content/suppl/2010/12/28/25.1.77.DC1
Material

References This article cites 43 articles, 14 of which can be accessed free at:

http://genesdev.cshlp.org/content/25/1/77.full.html\#ref-list-1

License

Email Alerting Receive free email alerts when new articles cite this article - sign up in the box at the top

Service right corner of the article or click here.

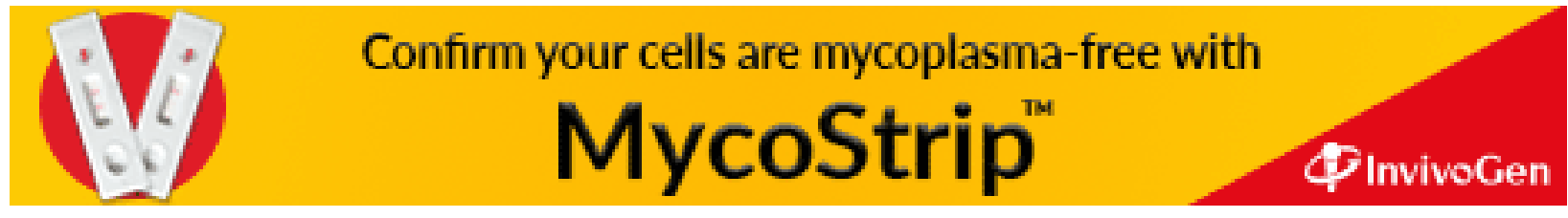

